\title{
Hadron tomography in meson-pair production and gravitational form factors
}

\author{
S. Kumano ${ }^{a, b}$, Qin-Tao Song ${ }^{* a}$, and O. V. Teryaev ${ }^{c}$ \\ ${ }^{a}$ KEK Theory Center, Institute of Particle and Nuclear Studies, KEK, \\ and Department of Particle and Nuclear Physics, Graduate University for Advanced Studies \\ (SOKENDAI), Ooho 1-1, Tsukuba, Ibaraki, 305-0801, Japan \\ ${ }^{b}$ J-PARC Branch, KEK Theory Center, Institute of Particle and Nuclear Studies, KEK, \\ and Theory Group, Particle and Nuclear Physics Division, J-PARC Center, \\ 203-1, Shirakata, Tokai, Ibaraki, 319-1106, Japan \\ ${ }^{c}$ Bogoliubov Laboratory of Theoretical Physics, Joint Institute for Nuclear Research, \\ 141980 Dubna, Russia
}

Generalized Parton Distributions (GPDs) are 3-Dimensional (3D) structure functions for hadrons, and they are important for solving the proton spin puzzle including partonic orbital-angularmomentum contributions. The $s-t$ crossed quantities of the GPDs are Generalized Distribution Amplitudes (GDAs). Here, $s$ and $t$ are Mandelstam variables. The GDAs can be studied in two-photon processes $\left(\gamma^{*} \gamma \rightarrow h \bar{h}\right)$ at KEKB. A GDA describes the amplitude from quark and antiquark to the hadron pair $h \bar{h}$. In 2016, the Belle collaboration reported measurements for pionpair production in electron-positron collision, and the pion GDAs were determined in this work by analyzing the Belle data. In our analysis, the pion GDAs are expressed by a few parameters, which are determined by analyzing the Belle data. From the obtained GDAs, form factors of energy-momentum tenor, so called gravitational form factors, are calculated for pion in the timelike region. The spacelike gravitational form factors are calculated from the timelike ones by using the dispersion relation. Then, the mass radius of pion is calculated as $0.32-0.39 \mathrm{fm}$ and the mechanical radius, defined by the slope of the form factor $\Theta_{1}$, is calculated as $0.82-0.88 \mathrm{fm}$ for the pion by using the spacelike form factors. This is the first study on gravitational form factors and radii of hadrons from actual experimental measurements. In 2019, the Belle II collaboration will start collecting data by the higher luminosity Super KEKB, so that the GDAs of other hadrons should also be investigated in the near future. Our studies are valuable in understanding $3 \mathrm{D}$ structure and gravitational properties of hadrons.

23rd international spine symposium (Spin 2018)

September 10-14, 2018

Ferrara, Italy

\footnotetext{
*Speaker.
} 


\section{Introduction}

In Deeply Virtual Compton Scattering (DVCS), Generalized Parton Distributions (GPDs) can be investigated. From the GPDs, one can obtain partonic orbital-angular-momentum contributions to the nucleon spin. Therefore, the GPDs are key quantities to solve the proton spin puzzle. The GPDs are one type of 3-Dimensional (3D) structure functions, and they reveal internal structure for hadrons. Their forward limit and moments are Parton Distribution Functions (PDFs) and spacelike form factors. Other 3D structure functions are Generalized Distribution Amplitudes (GDAs), which are the $s-t$ crossed quantities of the GPDs in the Mandelstam variables $s$ and $t$. The GDAs prove us information on timelike form factors and Distribution Amplitudes (DAs). They are also useful for understanding the GPDs by the $s-t$ crossing.

In order to find the GDAs, one may use the two-photon process $\gamma^{*} \gamma \rightarrow h \bar{h}[1,2,3,4,5]$ and $\gamma^{*} N \rightarrow h \bar{h} N$ [6]. In the latter reaction, both GPDs and GDAs are involved. However, the amplitude of $\gamma^{*} \gamma \rightarrow h \bar{h}$ is associated only with the GDAs, so that it is possible to find the GDAs from experimental measurements. The two-photon process can be measured in the $e^{+} e^{-}$collision at the KEK B factory, and the appropriate kinetics range should be chosen to satisfy a factorization condition for extracting the GDAs. In 2016, the Belle collaboration released measurements of differential cross sections for $\gamma^{*} \gamma \rightarrow \pi^{0} \pi^{0}$ [7]. One can obtain the pion GDAs by analyzing the Belle data [4, 5]. Moreover, timelike form factors can be calculated from the determined GDAs [5]. In 2018, the KEKB facility was updated with a higher luminosity of $810^{35} \mathrm{~cm}^{-2} \mathrm{~s}^{-1}$, and the Belle II collaboration started taking data. There will be precise data of $\gamma^{*} \gamma \rightarrow h \bar{h}$ for other hadrons, so the GDAs of other hadrons can be investigated as well.

\section{Pion GDAs in the process $\gamma^{*} \gamma \rightarrow \pi^{0} \pi^{0}$}

In the DVCS, its amplitude can be factorized into a soft part and a hard one, and the soft part of the amplitude is described by the GPDs. If we consider the $s-t$ crossed channel of DVCS, it is the two-photon process $\gamma^{*} \gamma \rightarrow h \bar{h}$ shown in Fig. 1. Similarly, the soft part of the two-photon process is described by the GDAs, and $Q^{2}=-q_{1}^{2}$ should be much larger than the center-of-mass energy $W^{2}=\left(p_{1}+p_{2}\right)^{2}$ to satisfy the factorization condition. The GDA describes the amplitude of a quark-antiquark pair to the hadron-antihadron pair. Here, we take $\pi^{0}$ as for the hadron $h$ and define the GDAs as $[1,2,3]$ :

$$
\Phi_{q}^{\pi^{0} \pi^{0}}\left(z, \zeta, W^{2}\right)=\left.\int \frac{d y^{-}}{2 \pi} e^{i(2 z-1) P^{+} y^{-} / 2}\left\langle\pi^{0}\left(p_{1}\right) \pi^{0}\left(p_{2}\right)\left|\bar{q}(-y / 2) \gamma^{+} q(y / 2)\right| 0\right\rangle\right|_{y^{+}=\vec{y}_{\perp}=0},
$$

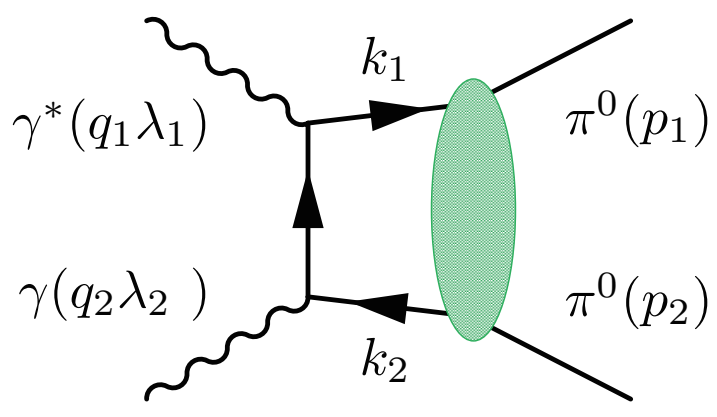

Figure 1: Two-photon process $\gamma^{*} \gamma \rightarrow \pi^{0} \pi^{0}$. The soft part is the pion GDA. 
where $P=p_{1}+p_{2}, z=k_{1}^{+} / P^{+}$is the momentum fraction of a quark, and $\xi=p_{1}^{+} / P^{+}$is the momentum fraction of the pion. In Fig. $1, \lambda_{1}$ and $\lambda_{2}$ are the helicities of the virtual photon and the real photon, respectively. We denote helicity amplitudes as $A_{\lambda_{1} \lambda_{2}}$, and there are three independent amplitudes $A_{++}, A_{0+}$ and $A_{+-}$by considering the parity invariance. The leading-twist term $A_{++}$ can be expressed by the pion GDA as [1,2]:

$$
A_{++}=\sum_{q} \frac{e_{q}^{2}}{2} \int_{0}^{1} d z \frac{2 z-1}{z(1-z)} \Phi_{q}^{\pi^{0} \pi^{0}}\left(z, \xi, W^{2}\right) .
$$

The amplitude $A_{0+}$ is a higher-twist term, and it is suppressed by $1 / Q$. The amplitude $A_{+-}$has an additional running coupling constant $\alpha_{s}\left(Q^{2}\right)$ since the gluon GDA is involved. Therefore, both $A_{0+}$ and $A_{+-}$can be neglected at large $Q^{2}$, and the differential cross section is expressed only by the amplitude $A_{++}$for $\gamma^{*} \gamma \rightarrow \pi^{0} \pi^{0}[1,2]$ as:

$$
d \sigma=\frac{1}{4} \alpha^{2} \pi \frac{\sqrt{1-\frac{4 m_{\pi}^{2}}{s}}}{Q^{2}+s}\left|A_{++}\right|^{2} \sin \theta d \theta,
$$

where $\alpha$ is the fine structure constant, and $\theta$ is the scattering angle in the c.m. frame of the final pions.

In the large $Q^{2}$ limit, the GDAs are independent of $Q^{2}$ and their asymptotic form [1,2] is given by

$$
\begin{aligned}
& \sum_{q} \Phi_{q}^{\pi^{0} \pi^{0}}\left(z, \xi, W^{2}\right)=18 n_{f} z(1-z)(2 z-1)\left[\tilde{B}_{10}(W)+\tilde{B}_{12}(W) P_{2}(\cos \theta)\right], \\
& \tilde{B}_{n l}(W)=\bar{B}_{n l}(W) \exp \left(i \delta_{l}\right), \quad \zeta=\frac{1+\beta \cos \theta}{2}, \beta=\sqrt{1-\frac{4 m_{\pi}^{2}}{s}}
\end{aligned}
$$

In Eq. (2.4), there are two terms, $\tilde{B}_{10}(W)$ for S-wave $\pi^{0} \pi^{0}$ and $\tilde{B}_{12}(W)$ for D-wave $\pi^{0} \pi^{0}$. The $\delta_{0}$ and $\delta_{2}$ are the S- and D-wave phase shifts [8,9], respectively, in the $\pi \pi$ elastic scattering below the $K K$ threshold. Above the $K K$ threshold, we introduced additional phase in the GDA analysis.

In the two-photon process, the resonance effect [6] is important and it is shown in Fig. 2. In the GDA analysis, we introduced $f_{0}(500)$ for an $\mathrm{S}$-wave resonance and $f_{0}(1270)$ for $\mathrm{D}$-wave resonance, whereas $f_{0}(980)$ is not considered in this analysis since it is not clearly shown in the differential cross section.

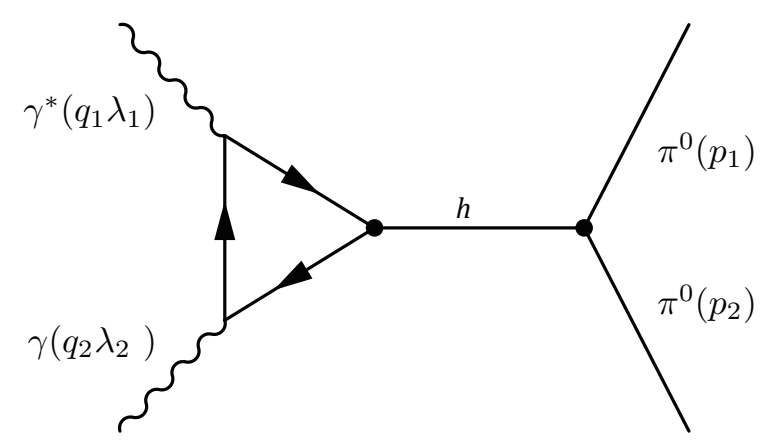

Figure 2: Resonance effect through the process $\gamma^{*} \gamma \rightarrow h \rightarrow \pi^{0} \pi^{0}$. 


\section{Pion GDA analysis of the Belle data}

In our analysis, the pion GDAs are expressed by the addition of continuum terms and resonance ones as $[4,5]$ :

$$
\begin{aligned}
& \Phi_{q}^{\pi^{0} \pi^{0}}\left(z, \xi, W^{2}\right)=N_{h} z^{\alpha}(1-z)^{\alpha}(2 z-1)\left[\tilde{B}_{10}(W)+\tilde{B}_{12}(W) P_{2}(\cos \theta)\right], \\
& \tilde{B}_{10}(W)=\left\{\frac{-3+\beta^{2}}{2} \frac{10 R_{\pi}}{9 n_{f}} F_{h}\left(W^{2}\right)+\frac{5 g_{f_{0} \pi \pi f_{f_{0}}}}{3 \sqrt{2} \sqrt{\left[\left(M_{f_{0}}^{2}-W^{2}\right)^{2}+\Gamma_{f_{0}}^{2} M_{f_{0}}^{2}\right]}}\right\} e^{i \delta_{0}}, \\
& \tilde{B}_{12}(W)=\left\{\beta^{2} \frac{10 R_{\pi}}{9 n_{f}} F_{h}\left(W^{2}\right)+\beta^{2} \frac{10 g_{f_{2} \pi \pi f_{2}} M_{f_{2}}^{2}}{9 \sqrt{2} \sqrt{\left(M_{f_{2}}^{2}-W^{2}\right)^{2}+\Gamma_{f_{2}}^{2} M_{f_{2}}^{2}}}\right\} e^{i \delta_{2}} .
\end{aligned}
$$

Here, the parameter $\alpha$ is predicted as $\alpha=1$ in the large $Q^{2}$ limit, and $R_{\pi}=0.5$ is the momentum fraction carried by quarks in the pion. We take $\delta_{0}$ and $\delta_{2}$ as the $\pi \pi$ scattering phase shifts in the isospin $=0$ channel $[8,9]$. The overall factor $N_{h}$ depends on $\alpha$ to satisfy the sum rule [3],

$$
\int_{0}^{1} d z(2 z-1) \Phi_{q}^{\pi^{0}} \pi^{0}(z, \zeta, 0)=-2 R_{\pi} \zeta(1-\zeta)
$$

The resonance effect of $f_{0}(500)$ appears in the S-wave term $\tilde{B}_{10}(W)$, and the D-wave term $\tilde{B}_{12}(W)$ contains the resonance effect of $f_{2}$ (1270). The details of Eq. (3.1) are explained in Ref. [5].

There are 5 parameters in our GDA expression, and they are determined by analyzing the Belle data. We show the differential cross section of $\gamma^{*} \gamma \rightarrow \pi^{0} \pi^{0}$ in comparison with Belle data in Fig. 3, and this GDA analysis gives a good description of the experimental data with $\chi^{2} /$ d.o.f. $=1.09$ [5]. In Fig. 3, the resonance peak of $f_{2}(1270)$ is seen at $W \simeq 1.2 \mathrm{GeV}$, and it is a dominant contribution at this invariant-mass region with the Breit-Wigner form of the resonance in Eq. (3.1). The resonance effect of $f_{0}(500)$ is a broad distribution due to the large decay width.
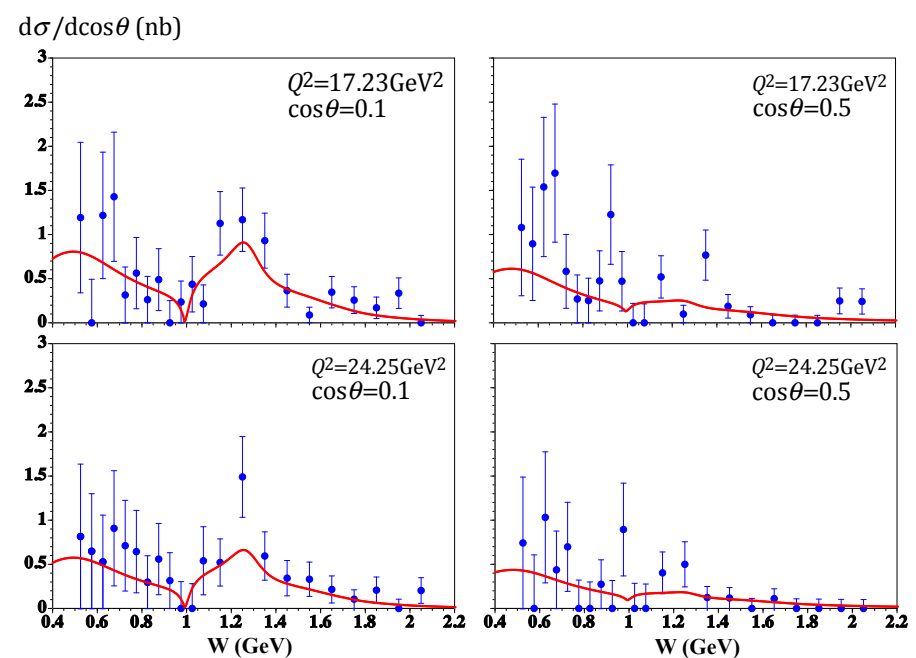

Figure 3: $W$ dependence of the cross section on $\gamma^{*} \gamma \rightarrow \pi^{0} \pi^{0}$ in comparison with Belle measurements. The energy scale $Q^{2}$ is set as $17.23 \mathrm{GeV}^{2}$ and $24.25 \mathrm{GeV}^{2}$, and $\cos \theta$ is set as 0.1 and 0.5 [5]. 
The hadronic matrix element of the energy-momentum tensor $T_{q}^{\mu v}$ can be expressed by the GPD in the spacelike region. Similarly, we can also study the matrix element of the energy-momentum tensor for pion in the timelike region by the pion GDA, and it is expressed as [3]:

$$
\int_{0}^{1} d z(2 z-1) \Phi_{q}^{\pi^{0} \pi^{0}}\left(z, \zeta, W^{2}\right)=\frac{2}{\left(P^{+}\right)^{2}}\left\langle\pi^{0}\left(p_{1}\right) \pi^{0}\left(p_{2}\right)\left|T_{q}^{++}(0)\right| 0\right\rangle .
$$

The right side of Eq. (3.3) can be expressed by two gravitational form factors $\Theta_{1}$ and $\Theta_{2}$ as:

$$
\left\langle\pi^{0}\left(p_{1}\right) \pi^{0}\left(p_{2}\right)\left|\sum_{q} T_{q}^{\mu v}(0)\right| 0\right\rangle=\frac{1}{2}\left[\left(s g^{\mu v}-P^{\mu} P^{v}\right) \Theta_{1}(s)+\Delta^{\mu} \Delta^{v} \Theta_{2}(s)\right],
$$

where $\Delta=p_{1}-p_{2}$. The form factor $\Theta_{1}$ is related to the mass or energy, and $\Theta_{2}$ is related to mechanical properties (pressure and shear force). The gravitational form factors of the pion can be obtained from the determined GDAs [5] as:

$$
\Theta_{1}(s)=\frac{3}{5}\left[\widetilde{B}_{12}\left(W^{2}\right)-2 \widetilde{B}_{10}\left(W^{2}\right)\right], \quad \Theta_{2}(s)=\frac{9}{5 \beta^{2}} \widetilde{B}_{12}\left(W^{2}\right) .
$$

In Eq. (3.5), $\Theta_{1}(s)$ contains both S-wave and D-wave contributions, and $\Theta_{2}(s)$ contains only the D-wave one. We show the timelike gravitational form factors $\Theta_{1}(s)$ and $\Theta_{2}(s)$ in the left panel of Fig. 4, and the resonance effect of $f_{2}(1270)$ is seen around $s=1.5 \mathrm{GeV}^{2}$ in both $\Theta_{1}$ and $\Theta_{2}$.

By the dispersion relation [10], the timelike gravitational form factors can be converted to the spacelike gravitational form factors:

$$
F^{h}(t)=\int_{4 m_{h}^{2}}^{\infty} \frac{d s}{\pi} \frac{\operatorname{Im} F^{h}(s)}{s-t-i \varepsilon} .
$$

We plot the normalized spacelike gravitational form factors $\Theta_{1}(t)$ and $\Theta_{2}(t)$ in the right panel of Fig. 4, and we find that $\Theta_{1}(t)$ decreases faster than $\Theta_{2}(t)$ as $|t|$ increases.
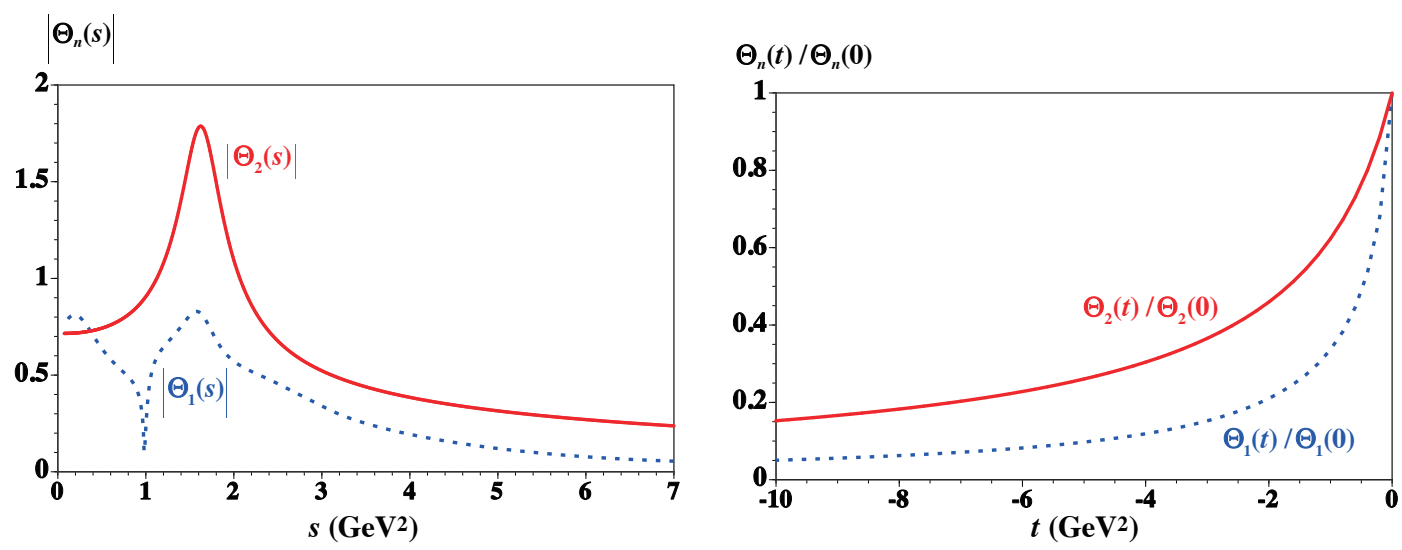

Figure 4: Left panel: The gravitational form factors $\Theta_{1}(s)$ and $\Theta_{2}(s)$ in the timelike region for pion. Right panel: The gravitational form factors $\Theta_{1}(t)$ and $\Theta_{2}(t)$ in the spacelike region for pion [5].

The root-mean-square (rms) radii can be obtained from the spacelike gravitational form factors $\Theta_{1}(t)$ and $\Theta_{2}(t)$ by calculating the slopes of gravitational form factors at $t=0$ :

$$
\left\langle r^{2}\right\rangle_{h}=\left.\frac{6}{F(t=0)} \frac{F^{h}(t)}{d t}\right|_{|t| \rightarrow 0}=\frac{6}{F(t=0)} \int_{4 m_{\pi}^{2}}^{\infty} d s \frac{\operatorname{Im} F^{h}(s)}{\pi s^{2}}, F(t=0)=\int_{4 m_{\pi}^{2}}^{\infty} d s \frac{\operatorname{Im} F^{h}(s)}{\pi s},
$$


where $F^{h}$ is $\Theta_{1}$ or $\Theta_{2}$. The gravitational radii are calculated as the mass radius $\sqrt{\left\langle r^{2}\right\rangle_{\text {mass }}}=0.39$ fm from $\Theta_{1}$ and the mechanical radius $\sqrt{\left\langle r^{2}\right\rangle_{\text {mech }}}=0.82 \mathrm{fm}$ from $\Theta_{2}$ [5].

In our analysis, $\delta_{0}$ and $\delta_{2}$ are the $\pi \pi$ scattering phase shifts below the $K K$ threshold, and we introduced the additional phase for $\mathrm{S}$-wave phase shift above the $K K$ threshold. However, even if we add the additional phase to D-wave phase shift above the threshold, the Belle data are equally-well explained. In this second analysis, we obtained the mass radius $\sqrt{\left\langle r^{2}\right\rangle_{\text {mass }}}=0.32 \mathrm{fm}$ and the mechanical radius $\sqrt{\left\langle r^{2}\right\rangle_{\text {mech }}}=0.88 \mathrm{fm}$. Therefore, the gravitational radii [5] are estimated as:

$$
\sqrt{\left\langle r^{2}\right\rangle_{\text {mass }}}=0.32 \sim 0.39 \mathrm{fm}, \sqrt{\left\langle r^{2}\right\rangle_{\text {mech }}}=0.82 \sim 0.88 \mathrm{fm},
$$

in our analyses. The charge radius of pion is $\sqrt{\left\langle r^{2}\right\rangle_{\text {charge }}}=0.672 \pm 0.008 \mathrm{fm}$ [11], so that the mass radius is smaller than the charge radius and the mechanical one is slightly larger. About progress on the gravitational form factors, one may also look at recent studies [12].

\section{Summary}

The GPDs are expected to solve the proton spin puzzle, because they can reveal partonic orbitalangular-momentum contributions. Moreover, the hadronic matrix elements of the energy-momentum tensor can also be studied by the GPDs, which provide us a good way to investigate the gravitational form factors since they cannot be probed experimentally by direct gravitational interactions. The GDAs are the $s-t$ crossed quantities of GPDs, so that the GDAs can be also used to study the GPDs and gravitational form factors. In this work, we analyzed the experimental data to obtain the GDAs of pion by using the KEKB measurements on the two-photon process $\gamma^{*} \gamma \rightarrow \pi^{0} \pi^{0}$. From the determined GDAs, the gravitational form factors were calculated. Furthermore, the gravitational radii were calculated for the pion, and we obtained the mass radius $(0.32-0.39 \mathrm{fm})$ and mechanical radius $(0.82-0.88 \mathrm{fm})$. In the near future, we expect that the Belle II collaboration will release much precise measurements for the process $\gamma^{*} \gamma \rightarrow h \bar{h}$ for various hadrons in addition to the $\pi^{0}$ pair. Then, those measurements will help us understand much detail on the hadron structure through the GDAs.

\section{References}

[1] M. Diehl, T. Gousset, B. Pire and O. Teryaev, Phys. Rev. Lett. 81 (1998) 1782.

[2] M. Diehl, T. Gousset and B. Pire, Phys. Rev. D 62 (2000) 073014.

[3] M. V. Polyakov, Nucl. Phys. B 555 (1999) 231.

[4] H. Kawamura and S. Kumano, Phys. Rev. D 89 (2014) 054007.

[5] S. Kumano, Q. T. Song and O. V. Teryaev, Phys. Rev. D 97 (2018) 014020. See the version 3 of arXiv: 1711.08088 for erratum, submitted to Phys. Rev. D.

[6] I. V. Anikin, B. Pire, L. Szymanowski, O. V. Teryaev and S. Wallon, Phys. Rev. D 71 (2005) 034021.

[7] M. Masuda et al. [Belle Collaboration], Phys. Rev. D 93 (2016) 032003.

[8] P. Bydzovsky, R. Kamiski and V. Nazari, Phys. Rev. D 90 (2014) 116005.

[9] V. Nazari, P. Bydzovsky, and R. Kaminski, Phys. Rev. D 94 (2016) 116013.

[10] See Sec. 18.8. in J. D. Bjorken and S. D. Drell, Relativistic Quantum Fields (McGraw-Hill, New York, 1965).

[11] C. Patrignani et al. [Particle Data Group], Chin. Phys. C 40 (2016) 100001.

[12] M. V. Polyakov and P. Schweitzer, arXiv:1801.05858 [hep-ph]; V. D. Burkert, L. Elouadrhiri and F. X. Girod, Nature 557 (2018) no.7705, 396; K. Tanaka, arXiv:1806.10591 [hep-ph]. 